936). (Respond: Dr K Otani, Department of Pediatric Neurology, Osaka Medical Center, 840 Murodo-cho, Izumi, Osaka 590-02, Japan).

COMMENT. The authors cite 4 reports including eight previous patients with secondary sensory seizures published in the last 40 years, the first involving 2 patients of Penfield and Jasper (1954). One study was entitled 'Sensory seizures mimicking a psychogenic seizure.' (Lessor RP et al. Neurology 1983;33:800). It is certainly conceivable that the diagnosis is sometimes overlooked and the symptoms misinterpreted as psychogenic.

\title{
EEG MONITORING OF NEONATAL SEIZURES
}

Sixty-three neonates were investigated using prolonged video/EEG monitoring to identify seizures and determine the diagnostic efficiency of clinical observation and short duration EEGs at the Department of Paediatric Neurology, Prince of Wales Children's Hospital, Sydney, NSW, Australia. Thirtytwo patients had seizures confirmed. Clinical observations after anticonvulsant treatment identified seizures in $66 \%$, and a $60 \mathrm{~min}$ EEG revealed electrographic seizures in $76 \%$, after phenobarbital treatment, and in $50 \%$ after addition of phenytoin. Short duration EEG avoids misdiagnoses in most patients with ambiguous clinical signs and aids substantially in the identification of neonatal seizures. (Bye A, Flanagan D. Electroencephalograms, clinical observations and the monitoring of neonatal seizures. I Paediatr Child Health December 1995;31:503-507). (Respond: Dr A Bye, Prince of Wales Children's Hospital, High St, Randwick, NSW 2031, Australia).

COMMENT. When clinical signs of seizures are controlled by anticonvulsants, a 60 min EEG is required to uncover subclinical neonatal seizures, and in some cases, especially when phenytoin has been given in addition to phenobarbital, prolonged video/EEG monitoring may be necessary in diagnosis. An EEG after infusion of anticonvulsant does not gaurantee seizure identification, but the probability of diagnosis increases in relation to the length of the recording. In a study at the Magee-Womens Hospital, Pittsburgh, PA, more than $50 \%$ of 92 neonates with seizures had only electrographic expression of seizures, and $16 \%$ exhibited electroclinical dissociation. (see Progress in Pediatric Neurology II, PNB Publishers, 1994, pp 11-16).

\section{DEGENERATIVE AND METABOLIC DISORDERS}

\section{CARNITINE DEFICIENCY SYNDROMES}

Carnitine deficiency syndromes are reviewed from the Departments of Neurology and Pediatrics, Columbia Presbyterian Medical Center, New York, NY. Primary carnitine deficiency is a decrease of intracellular carnitine that impairs fatty acid oxidation and is not associated with another systemic illness. It may be systemic or muscular, presenting as progressive cardiomyopathy, hypoketotic hypoglycemic encephalopathy, or myopathy. Age at onset is 1 month to 7 years, with a mean of 2 years. In encephalopathy, carnitine levels in plasma and tissues are below 10\% of normal, and acylcarnitines are proportionately reduced. Acylcarnitine to free carnitine ratio is normal. Treratment is oral carnitine at daily doses of 100 to $200 \mathrm{mg} / \mathrm{kg}$. Intermittent diarrhea and a fishy body odor are described as side effects of carnitine replacement. Muscle carnitine deficiency is characterized by severe reduction 